antibody, $28 \mathrm{H} 1$, to which the PS, IRDye700DX, for tPDT is attached. Here we investigated the feasibility of using FAP-tPDT to induce cell death in murine arthritic synovium ex vivo.

Methods: After conjugation of the IDRye700DX to $28 \mathrm{H} 1$ (28H1-700DX), binding and specificity of the conjugate was determined. Subsequently, tPDT efficiency in vitro was established using a 3 T3 fibroblast cell line stably transfected with FAP. Biodistribution using an $\left[{ }^{111} \mathrm{In}\right] \mathrm{In}-\mathrm{DTPA}-28 \mathrm{H} 1$ conjugate with and without IRDye700DX was performed in healthy C57BL/6N mice as well as in C57BL/6N mice with antigen induced arthritis (AIA). Finally, the potential of FAP-tPDT to induce targeted cell death in the synovial lining was determined by treating knee joints from mice with AIA ex vivo.

Results: Conjugation of IRDye700DX to the antibody did not negatively influence the immunoreactive fraction or binding capacity of the conjugate (94.7\% for $28 \mathrm{H} 1-700 \mathrm{DX})$. $28 \mathrm{H} 1-700 \mathrm{DX}$ was able to efficiently induce FAP-specific cell death in vitro. At $17.6 \mathrm{~J} / \mathrm{cm}^{2}$ radiant exposure, $89.24 \%$ $\pm 3.67 \%$ of fibroblasts died in the group incubated with antibody compared to control incubated with buffer only $(p<0.001)$. Biodistribution of the compound with the PS showed increased accumulation in the liver compared to the antibody without PS $(31.46 \pm 5.49 \%$ injected dose per gram tissue $(\% \mathrm{ID} / \mathrm{g})$ versus $5.32 \pm 1.17 \% \mathrm{ID} / \mathrm{g}$ for the antibody with or without PS, respectively $(p<0.001))$. However, despite this increased clearance to the liver, accumulation in the inflamed joints was increased in the group injected with the antibody-PS construct $(1.61 \pm 0.08 \%$ ID versus $1.13 \pm 0.06 \% \mathrm{ID}$ for the antibody with or without PS $(p<0.001))$. Interestingly, ex vivo FAP-tPDT of knee joints of arthritic mice caused significant photo-bleaching of the PS (19.69 $\pm 2.02 \%$ fluorescent signal remaining versus $96.00 \pm 25.98 \%$ compared to the unexposed control at baseline, $\mathrm{p}=0.047$ ). Furthermore FAP-tPDT induced marked apoptosis as was indicated by an increased staining of the markers caspase- 3 and yH2AX evident in the synovium of treated knee joints.

Conclusion: Here we demonstrated the feasibility of conjugating a PS to an antibody targeting FAP on activated SF without negatively impacting the binding capacity thereof. Furthermore we showed that this construct can then be used to deliver cell specific cytotoxicity through TPDT both in vitro and ex vivo in a mouse model of arthritis. This approach may have therapeutic potential in the treatment of RA.

Disclosure of Interests: Daphne Dorst: None declared, Mark Rijpkema: None declared, Mijke Buitinga: None declared, Peter Laverman: None declared, Marti Boss: None declared, Christian Klein Employee of: Roche pharmaceutics, Anne Freimoser-Grundschober Employee of: Roche pharmaceutics, Birgitte Walgreen: None declared, Peter van der Kraan: None declared, Martin Gotthardt: None declared, Marije Koenders: None declared

DOI: 10.1136/annrheumdis-2019-eular.7252

\section{SAT0053 \\ GLYCOSYLATION IN MAMMALS PROTECTS CITRULLINATED CHEMOKINES FROM PARTIAL DEGRADATION}

Olexandr Korchynskyi ${ }^{1,2,3} .{ }^{1}$ Rzeszow University, Medical Faculty, Rzeszow Poland; ${ }^{2}$ Institute of Cell Biology, Natl.Acad.Scie. of Ukraine, Lviv, Ukraine; ${ }^{3}$ Academic Medical Centre, University of Amsterdam, Amsterdam, Netherlands

Background: Citrullination is a postranslational modification of specific proteins by peptidylarginine deiminase (PAD) activities. Nowadays citrullination is recognized as a hallmark of rheutamoid arthritis and other autoimmune diseases. In our recent study we have shown a presence of citrullinated chemokines epithelial-derived monocyte chemotactic protein-1 (MCP-1/ CCL2), macrophage inflammatory protein-1 $\alpha$ (MIP-1 $\alpha / C C L 3)$ and neutrophil-activating peptide 78 (ENA-78/CXCL5) in biological fluids collected from patients suffering from rheumatoid arthritis (RA) versus non-autoimmune arthritis diseases and demonstrated citrullinated ENA-78/CXCL5 as an efficient macrophage chemoattractant in a contrast with non-citrullinated ENA-78/CXCL5. In our current work we found that citrullinated in vitro bacterially produced chemokines cannot be efficiently used as the standards in modified enzyme linked immunosorbent assay (ELISA) assays designed to detect citrullinated chemokines.

Objectives: In our work we aimed generate a procedure for preparation of stable citrullinated chemokines suitable for research aplications and to validate a hypothesis that posttranslational modifications occurring in mammalian cells that can stabilize chemokines can also protect citrullinated chemokines from quick degradation.

Methods: MCP-1/CCL2 and MIP-1 $\alpha /$ CCL3 were cloned from total RNA isolated from synovial fibroblasts obtained from RA patient. Both bacterially-produced and mammalian cells-produced recombinant human chemokines were citrullinated by commercial rabbit PAD2. Success in citrullination was confirmed with Western blotting and mass-spectrometry. Citrullinated chemokine concentrations were measured by modified sandwich ELISA assays.

Results: Both commercially available and self-made bacterially produced chemokines MCP-1/CCL2, MIP-1 $\alpha /$ CCL3 and ENA-78/CXCL5 undergo quick partial degradation upon their in vitro citrullination by PAD2 and cannot be detected with either Western blotting or mass-spectrometry. At the same time mammalian cells-produced properly glycosylated MCP-1/ CCL2, MIP-1 $\alpha /$ CCL3 and ENA-78/CXCL5 can be efficiently citrullinated and successfully used as the standards in modified ELISA assays as well as in bioassays.

Conclusion: Glycosylation that is lucking in bacterially-produced proteins but occurs in mammalian cells stabilizes citrullinated chemokines thus protecting them from quickly ongoing partial degradation.

BioEthics Committee Approval: All human subject samples were collected after approval by the Institutional Review Board of the Academic Medical Center/University of Amsterdam, Amsterdam, The Netherlands (Protocol MEC 07/079 \#10.17.0708) and provision of informed consent by the patients.

\section{REFERENCE}

[1] Yoshida K., Korchynskyi O., Tak P.-P., Isozaki T., Ruth J.H., Campbell P. L., Baeten D.L., Gerlag D.M., Amin M.A., and. Koch A.E. (2014) Arthritis and Rheumat 66, No 10, P.2716-2727.

Disclosure of Interests: None declared

DOI: 10.1136/annrheumdis-2019-eular.8072

\section{SAT0054 INVESTIGATING MECHANISMS OF AUTOANTIBODY INDUCED PAIN, BONE LOSS AND ARTHRITIS DEVELOPMENT}

Akilan Krishnamurthy ${ }^{1}$, Yogan Kisten ${ }^{1}$, Katalin Sandor $^{2}$, Alexandra Circiumaru ${ }^{1}$, Gustaf Wigerblad $^{1}$, Peter Damberg ${ }^{3}$, Koji Sakurabas ${ }^{1}$, Heidi Wähämaa ${ }^{3}$, Patrik Jarvolli ${ }^{4}$, Juan Jimenez Jimenez Andrade ${ }^{5}$, Vivianne Malmström ${ }^{1}$, Lars Klareskog ${ }^{1}$, Camilla Svensson ${ }^{2}$, Bence Réthi ${ }^{1}$, Anca Catrina ${ }^{1} .{ }^{1}$ Karolinska Institutet, Rheumatology unit, Medicine, stockholm, Sweden; ${ }^{2}$ Karolinska Institutet, Physiology and Pharmacology, stockholm, Sweden; ${ }^{3}$ Karolinska Institutet, Karolinska Experimental Research and Imaging Centre (KERIC), stockholm, Sweden; ${ }^{4}$ Karolinska Institutet, stockholm, Sweden; ${ }^{5}$ Universidad Autónoma de Tamaulipas, Reynosa, Tamaulipas, Mexico

Background: In rheumatoid arthritis (RA), autoantibodies against citrullinated proteins (ACPAs) have been reported to be associated with bone loss, pain and tenosynovitis prior to disease onset.

Objectives: We aimed to investigate if transfer of human ACPAs into mice could reproduce these clinical observations.

Methods: Monoclonal ACPA (1325:04C03 and 1325:01B09) and contro (1362:01E02) antibodies (mAbs) were generated from synovial plasma or memory B cells of RA patients. 2mg of combination of monoclonal ACPAs or control antibody were injected in BALB/c female mice (12-16 Weeks) with or without a consecutive intra-articular injection of LPS after 8 days. Pain-like behavior was monitored by measuring mechanical hypersensitivity using von Frey filaments every 3 days and estimation by up-down Dixon method. Bone mineral density was measured by microCT. Using specially designed mobilization casts, dedicated mouse MR coils, and gadolinium enhanced contrast medium, the hind limbs of these mice were scanned and evaluated for any signs of soft tissue joint inflammation. Blinded to ACPA and controls, the MRI images were scored for the presence of synovial thickening, effusion and tendon inflammatory changes by 3 readers in consensus.

Results: ACPAs (1325:04C03 and 1325:01B09) induced significantly more pronounced pain-like behavior (lasting for at least 4 weeks) and reduction of the trabecular bone thickness in the hind limbs, whereas no such effect was seen with the control mabs generated in the same way as the monoclonal ACPAs. While no macroscopic sign of joint inflammation could be detected, preliminary MRI data shows that sub-clinical joint inflammation (such as tenosynovitis) in mice injected with ACPAs but not those injected with control mAb. Intra-articular LPS injection resulted in significantly increased prolonged mechanical hypersensitivity in mice initially receiving sub-optimal doses of monoclonal ACPA as compared to those receiving control mAb. This was associated with higher levels of sub-clinical inflammation (as shown by MRI scans) in ACPA injected mice.

Conclusion: We show that ACPA induces pain-like behavior, bone loss and sub-clinical inflammation in mice, a model that mimics the pre-clinical state of ACPA positive RA. 


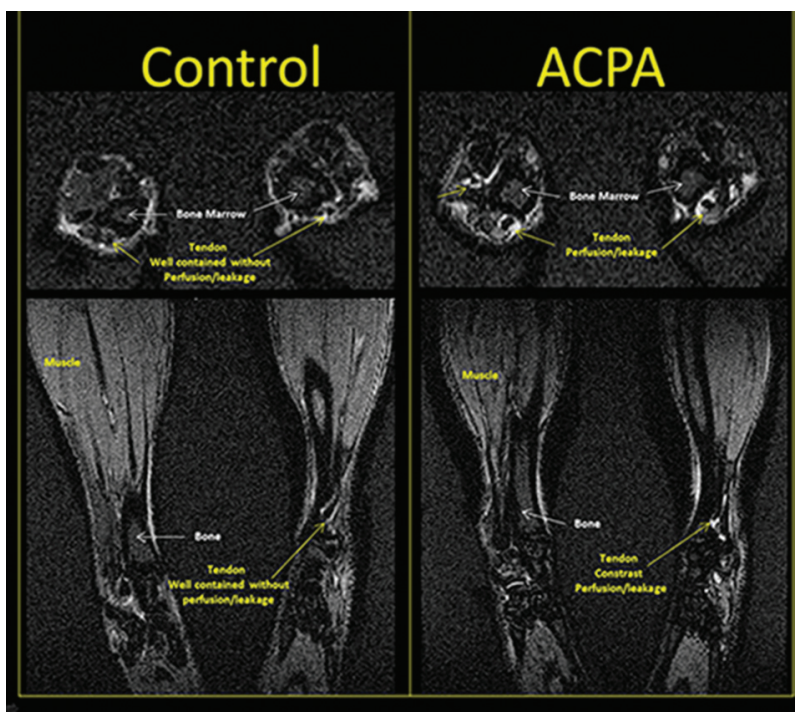

\section{REFERENCES}

[1] Harre, U. et al. J Clin Invest (2012)

[2] Krishnamurthy, A. et al. Ann Rheum Dis (2016)

[3] Wigerblad, G. et al. Ann Rheum Dis (2016)

[4] KleyerA, Seminars in Arthritis and Rheumatism (2016)

Disclosure of Interests: Akilan Krishnamurthy: None declared, Yogan Kisten: None declared, Katalin Sandor: None declared, Alexandra Circiumaru: None declared, Gustaf Wigerblad: None declared, Peter Damberg: None declared, Koji Sakurabas: None declared, Heidi Wähämaa: None declared, Patrik Jarvolli: None declared, Juan Jimenez Jimenez Andrade: None declared, Vivianne Malmström: None declared, Lars Klareskog Grant/ research support from: Yes, but not for the presented study., Camilla Svensson: None declared, Bence Réthi: None declared, Anca Catrina Grant/research support from: Yes, but not for the presented study. DOI: 10.1136/annrheumdis-2019-eular.5742

\section{SAT0055 JOINT SPECIFIC TNF RESPONSE OF SYNOVIAL FIBROBLASTS IN RHEUMATOID ARTHRITIS}

Raphael Micheroli ${ }^{1}$, Amanda Mcgovern ${ }^{2}$, Kerstin Klein $^{1}$, Xiangyu $\mathrm{Ge}^{2}$, Paul Martin ${ }^{2}$, Oliver Distler ${ }^{1}$, Mojca Frank-Bertoncelj ${ }^{1}$, Stephen Eyre ${ }^{2}$,

Caroline Ospelt'. ${ }^{1}$ Center of Experimental Rheumatology, Department of Rheumatology, University Hospital Zurich, Zurich, Switzerland; ${ }^{2}$ Arthritis Research UK Centre for Genetics and Genomics, School of Biological Sciences, Faculty of Biology, Medicine and Health, The University of Manchester, Manchester, United Kingdom

Background: Synovial fibroblasts (SF) in rheumatoid arthritis (RA) play a major role in chronic inflammation and joint destruction. We showed previously that their epigenetic and transcriptional profile as well as their function vary significantly between different joints. However, it is unknown whether there is a joint-specific inflammatory response of SF.

Objectives: To compare transcriptional changes between SF from hand, shoulder and knee joints after stimulation with the pro-inflammatory cytokine TNF.

Methods: We cultured SF from synovial tissues of hand (metacarpophalangeal and proximal interphalangeal joint), shoulder and knee joints of RA patients. After stimulation of the SF with $10 \mathrm{ng} / \mathrm{ul}$ TNF for $24 \mathrm{~h}(\mathrm{n}=2$ for each joint location), RNA was sequenced on the Illumina HiSeq4000 platform. We analyzed differential gene expression with $\mathrm{R} v 3.5 .2$ and CuffDiff and DESeq2 packages.

Results: As shown in Figure 1, principal component analysis showed evident separation of joint locations and condition (unstimulated vs TNF stimulated). In a sample-to-sample distance matrix, hand samples (TNF stimulated and unstimulated) grouped apart from shoulder and knee samples (Figure 2).

Of the regulated genes, $26 \%$ appeared in all three joint locations and $56 \%$ overlapped between knee and shoulder, but only 30\% overlapped between hand and shoulder and between hand and knee SF. Similarly, also enriched pathways differed particularly between hands and the more proximal joints.
'Defense response' $\left(p=8.11 \times 10^{-23}\right)$ and 'cytokine activity' $\left(p=1.27 \times 10^{-21}\right)$ were the most significantly enriched gene ontology terms for genes regulated in TNF stimulated hand SF. These processes were less prominently enriched in stimulated knee $\left(1.58 \times 10^{-15}\right.$ and $\left.8.84 \times 10^{-08}\right)$ and shoulder SF $\left(2.02 \times 10^{-11}\right.$ and $\left.3.20 \times 10^{-07}\right)$, where 'cell cycle' $\left(p=2.26 \times 10^{-30}\right.$ in knee and $\mathrm{p}=2.18 \times 10^{-32}$ in shoulder), and 'DNA packaging complex' $\left(\mathrm{p}=4.63 \times 10^{-49}\right.$ in knee and $p=8.91 \times 10^{-36}$ in shoulder) were the most significantly enriched gene ontology terms. These processes were not significantly enriched in stimulated hand SF.

Conclusion: SF from different joints in RA react differently to TNF stimulation. In particular hand SF reacted different to TNF stimulation than shoulder and knee SF, which appeared more similar. These qualitative and quantitative differences of the inflammatory response might translate into joint-specific pathotypes of synovitis with distinct therapeutic responses and disease outcomes.

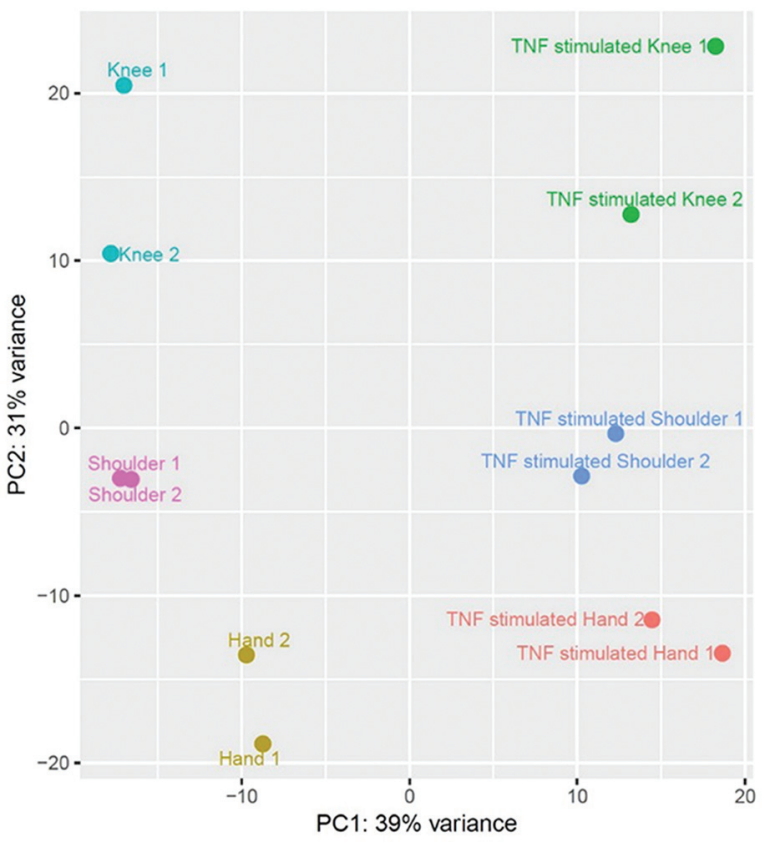

Figure 1. Principal component analysis of TNF stimulated and unstimulated synovial fibroblasts

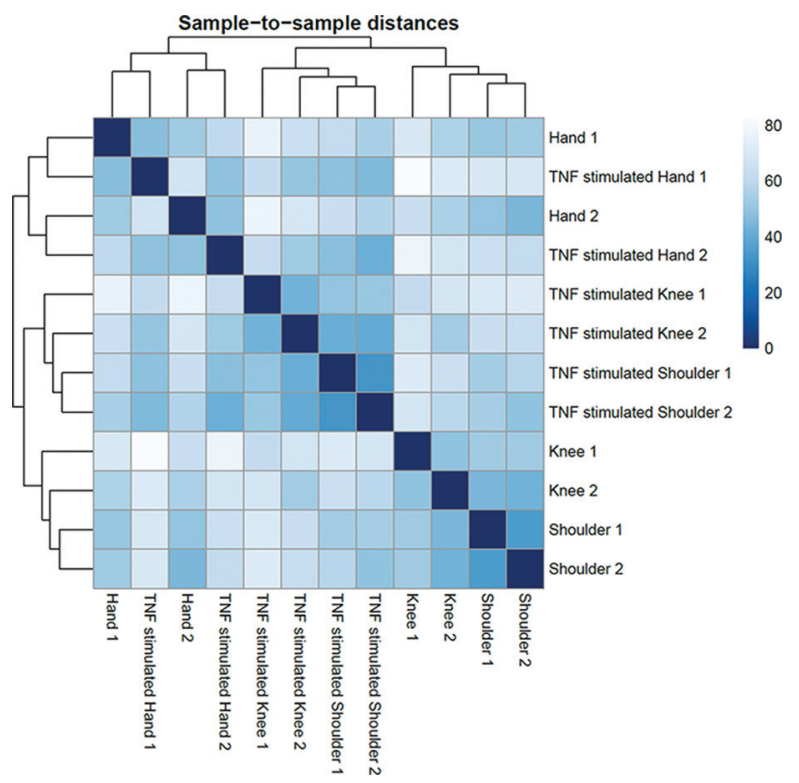

Figure 2. Sample-to-sample distance matrix of TNF stimulated and unstimulated synovial fibroblasts 